\title{
Modal Matching: An Approach to Natural Compliant Jumping Control
}

\author{
Dominic Lakatos ${ }^{1}$ and Alin Albu-Schäffer ${ }^{1,2}$
}

\begin{abstract}
This paper derives the basic concept of modal matching-an approach to natural motion control. Modal matching exploits the nonlinearity of the rigid multi-body dynamics (and the variability of the elastic transmissions) as degree of freedom to fit the natural plant dynamics to the desired dynamics of the task. Modal matching achieves a desired intrinsic oscillation behavior which is locally equivalent to the dynamics of the basic spring loaded inverted pendulum or pogo-stick model (both implementing a linear inertia acting on a linear spring), well established in locomotion analysis and control. Using the concept of modal matching, an efficient and effective methodology to natural jumping control is introduced.
\end{abstract}

\section{INTRODUCTION}

Physical compliance is a key feature of legged locomotion in robotic and biological systems [1], [2]. The elastic energy storages can be exploited to overcome peak power and velocity limitations [3], [4], [5], [6] on the one hand and improve the mechanical robustness [7], [8] on the other hand. Especially in case of cyclic locomotion tasks such as jumping, hopping or running, transferring resonance concepts from single mass-spring-damper systems to nonlinear elastic multi-body systems seems to be a key point of performance and energy efficiency. Very basic concepts in locomotion exploiting elasticities for the bouncing motion use either translational actuators [9] or translational spring models [10], [11], [12], [13]. These concepts naturally inherit the resonance properties of the linear spring-mass model. To implement such a desired behavior on real robots with segmented legs, approaches based on virtual model control [14], virtual constraints [15], or operational space control [16] have been successfully applied.

Our goal is to directly excite the plant in a resonance mode, while simultaneously obtaining the desired bouncing motion required for the specific gait. For simple motions such as vertical jumping, this can be achieved by our recent method proposed in [17]. The functional principle of the approach in [17] is to initially excite the system with a guess of the oscillation mode and observe the resulting motion. Based on this observation, the excitation is improved successively. The system converges to an excitation, respectively motion which correspond to an average of the eigenmode of the instantaneously linearized system. Since the resulting motion is an intrinsic property of the plant, it is potentially efficient,

\footnotetext{
${ }^{1}$ D. Lakatos and A. Albu-Schäffer are with the Robotic Mechatronic Center (RMC), Institute of Robotics and Mechatronics, German Aerospace Center (DLR), D-82234 Oberpfaffenhofen, Germany dominic.lakatosedlr. de

${ }^{2}$ A. Albu-Schäffer is also with Technical University Munich, Chair of Sensor Based Robots and Intelligent Assistance Systems, Department of Informatics, D-85748 Garching, Germany alin.albu-schaeffer@dlr.de
}

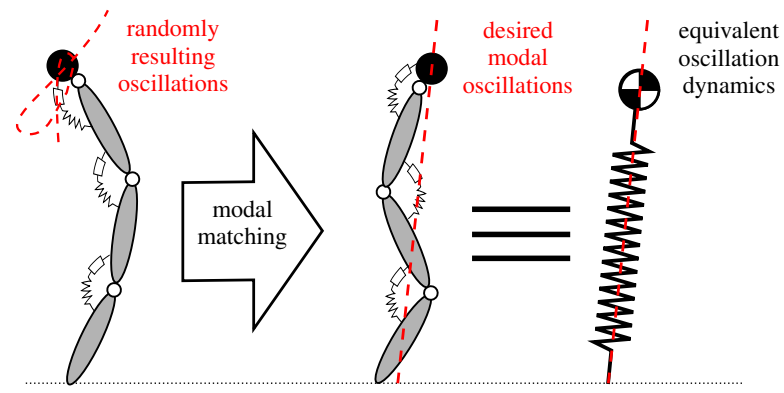

Fig. 1. The concept of modal matching.

but does not necessarily match to the desired task, which is given for example by a predefined forward jumping direction. In our follow up work [18], we proposed an approach which can be used to excite an oscillation in a direction specified by the task. Since this predefined oscillation mode is in general not the intrinsic behavior of the plant, the motion is performant (the task is achieved), but not necessarily efficient.

This paper presents a methodology which combines both requirements: task performance and efficiency. The approach adapts the intrinsic oscillatory behavior of the plant to the desired behavior of the task. This, is achieved by a novel concept which we call modal matching - an approach to natural motion control. An implication of modal matching is the generalization of the locomotion principles such as Raibert's pogo-stick [9] or the spring loaded inverted pendulum (SLIP) [10] to elastic, segmented multi-body legs dynamics, without the need of modifying the intrinsic dynamics behavior of the plant, for example by decoupling control. An intermediate result of the modal matching algorithm is an adaptation law to adjust the direction of the oscillation mode in a modally compatible manner. This property can be used to perform the transition from the landing phase/direction and push-off phase/direction in the stance phase of a directed, natural jumping motion.

The contributions of this paper are as follows:

- a formulation of the stance phase dynamics which prepares for the modal matching theory (Sect. III);

- the main result: modal matching theory and algorithm (basic concepts, Sect. II and formal theory, Sect. IV);

- a natural jumping control methodology directly derived from the modal matching theory (Sect. V).

\section{BASIC CONCEPTS}

This section explains the basic idea of modal matching and its application to natural jumping control. 
The class of systems considered here are compliantly actuated robots which are schematically depicted in Fig. 1 (left). In each joint, the inertia of a motor is connected via a elastic transmission (spring) to the succeeding link. This actuator principle is also depicted in Fig. 2. If the motors are held in a constant position and the links are deflected (e.g., according to an impulsive contact force), one can observe an oscillatory motion of the links. Due to the nonlinearity of the multi-body dynamics, the resulting oscillation will be also not linear (Fig. 1, left). The nonlinear oscillation is depending on the configuration. The basic idea of modal matching is to modify the mode by changing the configuration. The main tool of the modal matching algorithm is to compute how a change in the robot configuration changes its oscillatory behavior. This so-called differential mapping can be obtained by considerations of differential geometry. It is worth mentioning that a desired oscillation could be an oscillation which has no rotational components, i.e., an oscillation which is exclusively translational (Fig. 1, middle). Thereby, the resulting oscillation dynamics is (locally) equivalent to the oscillation dynamics of the simple spring-mass or pogostick model ${ }^{1}$ (Fig. 1, right). This is of major importance for the jumping control explained in Sect. V.

The development from the modal matching concept to natural jumping control can be performed by addressing the following subtasks:

1) landing and spring compression (energy storage) along the modal direction;

2) tilting the mode to achieve the transition from the compression phase to the push-off phase;

3) imposing an equilibrium position for the modal inclination, i. e., stabilizing the modal tilting motion.

1) can be achieved by matching the oscillation mode with the landing direction. 2) can be the result of a (positive) difference between the direction of the adjusted oscillation mode and the landing direction, which produces a force tilting the oscillation mode in the push-off direction. 3) can be achieved by analyzing the Jacobian corresponding to the differential mapping required for the modal matching algorithm, which can be used to derive a stabilizing potential for the modal inclination. 3) is required, since the modal tilting dynamics, essentially an inverted pendulum dynamics, is intrinsically unstable and thus very sensitive to disturbances.

\section{MODEL}

The considered model has two finite states, either the rigid body dynamics is completely free floating (flight phase) or all legs are in contact (stance phase). We start by describing the free floating dynamics and then introduce the constraints and the resulting dynamics imposed by the legs in contact. Even though the concepts are introduced based on simple examples, the model and the following theory will be formulated in a general way which fits to a single leg as well as multi-

\footnotetext{
${ }^{1}$ The equivalence is only in the modally matched direction approximately valid.
}

legged robots with fully-actuated and over-actuated ${ }^{2}$ stance phase dynamics. In particular, the constraints of the stance phase dynamics are not assumed to be explicitly solvable for the dependent configuration variables.

\section{A. Free floating dynamics}

The free floating dynamics may be partitioned in the form

$$
\left[\begin{array}{cc}
\boldsymbol{M}_{\mathrm{bb}}(\boldsymbol{q}) & \boldsymbol{M}_{\mathrm{bj}}(\boldsymbol{q}) \\
\boldsymbol{M}_{\mathrm{bj}}(\boldsymbol{q})^{T} & \boldsymbol{M}_{\mathrm{jj}}(\boldsymbol{q})
\end{array}\right]\left[\begin{array}{c}
\ddot{\boldsymbol{q}}_{\mathrm{b}} \\
\ddot{\boldsymbol{q}}_{\mathrm{j}}
\end{array}\right]+\left[\begin{array}{c}
\boldsymbol{b}_{\mathrm{b}}(\boldsymbol{q}, \dot{\boldsymbol{q}}) \\
\boldsymbol{b}_{\mathrm{j}}(\boldsymbol{q}, \dot{\boldsymbol{q}})
\end{array}\right]=\left[\begin{array}{c}
\mathbf{0} \\
\boldsymbol{\tau}_{\mathrm{j}}
\end{array}\right]
$$

Herein, the generalized coordinates $\boldsymbol{q}=\left(\boldsymbol{q}_{\mathrm{b}}^{T}, \boldsymbol{q}_{\mathrm{j}}^{T}\right)^{T}$ are subdivided into base coordinates $\boldsymbol{q}_{\mathrm{b}} \in \mathbb{R}^{n_{\mathrm{b}}}$ and joint coordinates $\boldsymbol{q}_{\mathrm{j}} \in \mathbb{R}^{n_{\mathrm{j}}}$ describing the base and joint configuration, respectively. The block matrices $M_{k l}$ represent the generalized inertia tensor and the bias forces $\boldsymbol{b}_{k}$ summarize generalized Coriolis/centrifugal and gravitational forces, for $k, l \in\{\mathrm{b}, \mathrm{j}\}$.

\section{B. Actuator dynamics}

The joint torques $\boldsymbol{\tau}_{\mathrm{j}}\left(\boldsymbol{\theta}, \boldsymbol{q}_{\mathrm{j}}\right) \in \mathbb{R}^{n_{\mathrm{j}}}$ in (1) are derived from the elastic potential $U\left(\boldsymbol{\theta}, \boldsymbol{q}_{\mathrm{j}}\right)$, i. e.,

$$
\boldsymbol{\tau}_{\mathrm{j}}\left(\boldsymbol{\theta}, \boldsymbol{q}_{\mathrm{j}}\right):=-\frac{\partial U\left(\boldsymbol{\theta}, \boldsymbol{q}_{\mathrm{j}}\right)}{\partial \boldsymbol{q}_{\mathrm{j}}},
$$

where $\boldsymbol{\theta} \in \mathbb{R}^{n_{j}}$ are generalized motor coordinates. Note that the potential $U\left(\boldsymbol{\theta}, \boldsymbol{q}_{\mathrm{j}}\right)$ itself is assumed to be positive definite in a sense that $U\left(\boldsymbol{\theta}, \boldsymbol{q}_{\mathbf{j}}\right)>0, \forall(\boldsymbol{\theta}-\boldsymbol{q})>\mathbf{0}$, and $U\left(\boldsymbol{\theta}, \boldsymbol{q}_{\mathbf{j}}\right)=0$ only if $(\boldsymbol{\theta}-\boldsymbol{q})=\mathbf{0}$. In particular, the Hessian of the potential $\boldsymbol{K}\left(\boldsymbol{\theta}, \boldsymbol{q}_{\mathrm{j}}\right):=\frac{\partial^{2} U\left(\boldsymbol{\theta}, \boldsymbol{q}_{\mathrm{j}}\right)}{\partial \boldsymbol{q}_{\mathrm{j}}{ }^{2}}$ referred to as the stiffness matrix is positive definite.

Following common simplifications [19] fully justified for many robotic systems, the actuator dynamics can be represented by $\boldsymbol{B} \ddot{\boldsymbol{\theta}}+\boldsymbol{\tau}_{\mathrm{j}}\left(\boldsymbol{\theta}-\boldsymbol{q}_{\mathrm{j}}\right)=\boldsymbol{\tau}_{\mathrm{m}}$, where $\boldsymbol{B} \in \mathbb{R}^{n_{\mathrm{j}} \times n_{\mathrm{j}}}$ is a constant, positive definite, and diagonal inertia matrix and $\tau_{\mathrm{m}} \in \mathbb{R}^{n_{j}}$ are generalized motor forces which are considered as control input. Note that the states $\boldsymbol{q}_{\mathrm{j}}, \dot{\boldsymbol{q}}_{\mathrm{j}}$ of the rigid body dynamics (1) are only indirectly actuated via the actuator dynamics $\boldsymbol{B} \ddot{\boldsymbol{\theta}}+\boldsymbol{\tau}_{\mathrm{j}}\left(\boldsymbol{\theta}-\boldsymbol{q}_{\mathrm{j}}\right)=\boldsymbol{\tau}_{\mathrm{m}}$, where the coupling is due to the generalized elastic forces introduced in (2). Using a PD control $\boldsymbol{\tau}_{\mathrm{m}}=-\boldsymbol{K}_{\mathrm{P}}\left(\boldsymbol{\theta}-\boldsymbol{\theta}_{\mathrm{des}}\right)-\boldsymbol{K}_{\mathrm{D}} \dot{\boldsymbol{\theta}}$ for the desired motor position $\boldsymbol{\theta}_{\text {des }}$, with high positive definite gain matrices $\boldsymbol{K}_{\mathrm{P}}, \boldsymbol{K}_{\mathrm{D}} \in \mathbb{R}^{n_{\mathrm{j}} \times n_{\mathrm{j}}}$ such that $\epsilon\left(\boldsymbol{B} \ddot{\boldsymbol{\theta}}+\boldsymbol{K}_{\mathrm{D}} \dot{\boldsymbol{\theta}}+\boldsymbol{\tau}_{\mathrm{j}}\right)=\boldsymbol{\theta}_{\text {des }}-$ $\boldsymbol{\theta} \approx \mathbf{0}$, we can approximately consider $\boldsymbol{\theta}$ in (2) respectively (1) as control input. This is as the singular perturbation assumption [20], $\epsilon=1 /\left\|\boldsymbol{K}_{\mathrm{P}}\right\| \rightarrow 0$, holds.

\section{Constrained dynamics}

The stance phase dynamics is modeled by incorporating the constraints

$$
\phi=\phi\left(\boldsymbol{q}_{\mathrm{b}}, \boldsymbol{d}, \boldsymbol{q}_{\mathrm{j}}\right) \in \mathbb{R}^{n_{\mathrm{j}}}=\mathbf{0}
$$

\footnotetext{
${ }^{2}$ The rigid body dynamics is fully-actuated if the number of kinematics degrees of freedom equals the number of actuator degrees of freedom. It is over-actuated if the latter exceed the former.
} 
into the free floating dynamics (1), i.e.,

$$
\begin{aligned}
\boldsymbol{\Gamma}_{\mathrm{b}}(\boldsymbol{q}, \dot{\boldsymbol{q}}, \ddot{\boldsymbol{q}}) & =\left(\frac{\partial \phi\left(\boldsymbol{q}_{\mathrm{b}}, \boldsymbol{d}, \boldsymbol{q}_{\mathrm{j}}\right)}{\partial \boldsymbol{q}_{\mathrm{b}}}\right)^{T} \boldsymbol{\lambda} \\
\mathbf{0} & =\left(\frac{\partial \phi\left(\boldsymbol{q}_{\mathrm{b}}, \boldsymbol{d}, \boldsymbol{q}_{\mathrm{j}}\right)}{\partial \boldsymbol{d}}\right)^{T} \boldsymbol{\lambda} \\
\boldsymbol{\Gamma}_{\mathrm{j}}(\boldsymbol{q}, \dot{\boldsymbol{q}}, \ddot{\boldsymbol{q}}) & =\left(\frac{\partial \phi\left(\boldsymbol{q}_{\mathrm{b}}, \boldsymbol{d}, \boldsymbol{q}_{\mathrm{j}}\right)}{\partial \boldsymbol{q}_{\mathrm{j}}}\right)^{T} \boldsymbol{\lambda}+\boldsymbol{\tau}_{\mathrm{j}}\left(\boldsymbol{\theta}, \boldsymbol{q}_{\mathrm{j}}\right)
\end{aligned}
$$

where for the free floating inverse dynamics, the following abbreviations are introduced: $\boldsymbol{\Gamma}_{\mathrm{b}}(\boldsymbol{q}, \dot{\boldsymbol{q}}, \ddot{\boldsymbol{q}}):=\boldsymbol{M}_{\mathrm{bb}}(\boldsymbol{q}) \ddot{\boldsymbol{q}}_{\mathrm{b}}+$ $\boldsymbol{M}_{\mathrm{bj}}(\boldsymbol{q}) \ddot{\boldsymbol{q}}_{\mathrm{j}}+\boldsymbol{b}_{\mathrm{b}}(\boldsymbol{q}, \dot{\boldsymbol{q}})$ and $\boldsymbol{\Gamma}_{\mathrm{j}}(\boldsymbol{q}, \dot{\boldsymbol{q}}, \ddot{\boldsymbol{q}}):=\boldsymbol{M}_{\mathrm{bj}}(\boldsymbol{q})^{T} \ddot{\boldsymbol{q}}_{\mathrm{b}}+$ $\boldsymbol{M}_{\mathrm{jj}}(\boldsymbol{q}) \ddot{\boldsymbol{q}}_{\mathrm{j}}+\boldsymbol{b}_{\mathrm{j}}(\boldsymbol{q}, \dot{\boldsymbol{q}})$. The constraints (3) are formulated such that the absolute positions of the contact points have to be constant during stance phase. To explicitly resolve the problem of over-actuation, the "artificial" coordinates $\boldsymbol{d} \in \mathbb{R}^{n_{\mathrm{j}}-n_{\mathrm{b}}}$ satisfying $\operatorname{dim}\left(\boldsymbol{q}_{\mathrm{b}}\right)+\operatorname{dim}(\boldsymbol{d})=\operatorname{dim}\left(\boldsymbol{q}_{\mathrm{j}}\right)$ are introduced. The introduction of the coordinates $\boldsymbol{d}$ "relaxes" $n_{\mathrm{j}}-n_{\mathrm{b}}$ constraints. Since during stance phase, these constraints are still active the corresponding force along these coordinates $\boldsymbol{\tau}_{\mathrm{d}}=\left(\partial \boldsymbol{q}_{\mathrm{j}} / \partial \boldsymbol{d}\right)^{T} \boldsymbol{\tau}_{\mathrm{j}}$ can be controlled to remove internal tension forces.

The Lagrange multipliers $\lambda$ in (4)-(6) can be eliminated by substituting (6) into (4), (5) yielding

$$
\begin{aligned}
\boldsymbol{\Gamma}_{\mathrm{b}} & =\left(\frac{\partial \phi}{\partial \boldsymbol{q}_{\mathrm{b}}}\right)^{T}\left(\frac{\partial \phi}{\partial \boldsymbol{q}_{\mathrm{j}}}\right)^{-T}\left(\boldsymbol{\Gamma}_{\mathrm{j}}-\boldsymbol{\tau}_{\mathrm{j}}\right) \\
\mathbf{0} & =\left(\frac{\partial \phi}{\partial \boldsymbol{d}}\right)^{T}\left(\frac{\partial \phi}{\partial \boldsymbol{q}_{\mathrm{j}}}\right)^{-T}\left(\boldsymbol{\Gamma}_{\mathrm{j}}-\boldsymbol{\tau}_{\mathrm{j}}\right)
\end{aligned}
$$

where for compactness of notation, the dependencies are omitted. Considering the second time derivative of (3), the constrained dynamics (7) and (8) can be written in component form (structurally equal to (1)):

$$
\overline{\boldsymbol{M}}\left[\begin{array}{c}
\ddot{q}_{\mathrm{b}} \\
\ddot{\boldsymbol{d}}
\end{array}\right]+\left[\begin{array}{l}
\overline{\boldsymbol{b}}_{\mathrm{b}} \\
\overline{\boldsymbol{b}}_{\mathrm{d}}
\end{array}\right]=\left[\begin{array}{l}
\boldsymbol{\Phi}_{\mathrm{jb}}^{-T} \\
\boldsymbol{\Phi}_{\mathrm{jd}}^{-T}
\end{array}\right] \tau_{\mathrm{j}}
$$

where explicit expressions for the inertia matrix $\bar{M}$ and the Jacobian matrices $\boldsymbol{\Phi}_{\mathrm{jb}}, \boldsymbol{\Phi}_{\mathrm{jd}}$ required for later computations are provided in the Appendix I.

\section{THEORY AND ALGORITHM}

This section derives the basic result, i. e., the theory and the algorithm of what we call modal matching. First, we introduce a representation of the oscillation modes which corresponds to the eigenmodes of the linearization of the constraint dynamics (9), Sect. III-C. Second, we derive the corresponding algorithm which matches the actual mode with the desired oscillation mode. At this point, it is worth mentioning that although the representation of the oscillation mode is based on a linearization, the algorithm exploits the nonlinearity of the dynamics (i.e., the dependency of the generalized inertia and stiffness on the configuration) as degree of freedom to adjust the eigenmode. In particular, the approach applies only to a nonlinear plant (i. e., a multi-body dynamics with configuration dependent inertia matrix).

\section{A. Linearized dynamics}

In the following, we describe the modal oscillation behavior of the stance phase dynamics, when the motor coordinates $\boldsymbol{\theta}$ are held constant. Let us therefore denote the task coordinates by $\boldsymbol{x}=\left(\boldsymbol{q}_{\mathrm{b}}^{T}, \boldsymbol{d}^{T}\right)^{T}$ and the task and configuration variables by $\boldsymbol{z}=\left(\boldsymbol{x}^{T}, \boldsymbol{q}_{\mathrm{j}}^{T}\right)^{T}$. Then, consider the linearization of (9): Sect. III-C

$$
\overline{\boldsymbol{M}}(\boldsymbol{z}) \ddot{\boldsymbol{x}}+\overline{\boldsymbol{K}}(\boldsymbol{z}) \delta \boldsymbol{x}_{\boldsymbol{q}_{\mathrm{j}}}=\overline{\boldsymbol{K}}(\boldsymbol{z}) \delta \boldsymbol{x}_{\boldsymbol{\theta}}
$$

where explicit expressions of the components of the inertia and stiffness matrix in task coordinates $\bar{M}(\boldsymbol{z})$ and $\overline{\boldsymbol{K}}(\boldsymbol{z})$, respectively, are provided in the Appendix I. In (10), the nonlinear elastic torques given by (2), Sect. III-B, are linearized based on a first order Taylor expansion with deflections $\delta \boldsymbol{x}_{\boldsymbol{q}_{\mathrm{j}}}=\left(\frac{\partial \phi}{\partial \boldsymbol{q}_{\mathrm{j}}}\right)^{-1} \frac{\partial \boldsymbol{\phi}}{\partial \boldsymbol{x}} \delta \boldsymbol{q}_{\mathrm{j}}, \delta \boldsymbol{x}_{\boldsymbol{\theta}}=\left(\frac{\partial \phi}{\partial \boldsymbol{q}_{\mathrm{j}}}\right)^{-1} \frac{\partial \phi}{\partial \boldsymbol{x}} \delta \boldsymbol{\theta}$. Note that in general the stiffness matrix $\overline{\boldsymbol{K}}(\boldsymbol{z})$ also depends on the motor coordinates $\boldsymbol{\theta}$, but since we are interested in the passive behavior (i.e., $\boldsymbol{\theta}=$ const.), this dependency drops out.

\section{B. Definition of modal matching}

On the basis of the linearized task dynamics (10), the representation of the oscillation mode results from the generalized eigenvalue problem

$$
\begin{aligned}
\lambda \overline{\boldsymbol{M}} \boldsymbol{w} & =\overline{\boldsymbol{K}} \boldsymbol{w} \\
\text { s.t. } \quad \boldsymbol{w}^{T} \boldsymbol{w} & =1,
\end{aligned}
$$

where $\lambda>0$ is a generalized eigenvalue and $\boldsymbol{w} \in \mathbb{R}^{n_{\mathrm{j}}}$ is a generalized eigenvector. We assume algebraic and geometric multiplicity of one, therefore the solution of problem (11), (12) results in $n_{\mathrm{j}}$ eigenvalues $\lambda_{i}$ and corresponding eigenvectors $\boldsymbol{w}_{i}$. In the following, we always only consider the eigenvector $\boldsymbol{w}_{k}$ which has minimum distance to the desired eigenvector $\boldsymbol{w}_{\text {des }}$, where $k=\min \left(\left\|\boldsymbol{w}_{i}-\boldsymbol{w}_{\text {des }}\right\|\right)$ and for simplicity of notation we define $\stackrel{i}{\boldsymbol{w}}:=\boldsymbol{w}_{k}$. The components of the generalized eigenvector $w_{i}$ describe the distribution of the oscillation amplitudes w.r.t. coordinate directions $x_{i}$ for a motion along the corresponding oscillation mode in the vicinity of $\boldsymbol{q}_{\mathrm{j}}=\boldsymbol{\theta}$. Thus, we refer to $\boldsymbol{w}$ as oscillation mode or simply mode. The process of finding a joint configuration $\boldsymbol{q}_{\mathrm{j}}$ such that $\boldsymbol{w}=\boldsymbol{w}_{\text {des }}$ is what we call modal matching.

\section{Modal matching algorithm}

The idea of the modal matching algorithm considers the differential mapping

$$
\mathrm{d} \boldsymbol{w}=\frac{\partial \boldsymbol{w}(\boldsymbol{z})}{\partial \boldsymbol{z}} \mathrm{d} \boldsymbol{z}
$$

and approximates $\mathrm{d} \boldsymbol{w} \approx \boldsymbol{w}_{\mathrm{des}}-\boldsymbol{w}$ and $\mathrm{d} \boldsymbol{z} \approx \boldsymbol{z}_{k+1}-\boldsymbol{z}_{k}$ such that the recursion

$$
\boldsymbol{z}_{k+1}=\boldsymbol{z}_{k}+\left[\frac{\partial \boldsymbol{w}}{\partial \boldsymbol{z}}\left(\boldsymbol{z}_{k}\right)\right]^{+}\left(\boldsymbol{w}_{\mathrm{des}}-\boldsymbol{w}\right)
$$

converges to a $\boldsymbol{z}_{\infty}$ which minimizes the error $\tilde{\boldsymbol{w}}=\boldsymbol{w}_{\mathrm{des}}-\boldsymbol{w}$. In (14), the operator $(\cdot)^{+}$denotes the generalized inverse of a matrix which is required since the linear system of equations (13) is under-determined, i. e., $\partial \boldsymbol{w} / \partial \boldsymbol{z}$ is a $n_{\mathrm{j}} \times 2 n_{\mathrm{j}}$ matrix. 
In particular, the matrix $\partial \boldsymbol{w} / \partial \boldsymbol{z}$ has row rank $n_{\mathrm{j}}-1$ since the length of the eigenvector is identity (cf. condition (12)). This rank-deficiency can be overcome by a transformation of the form $\boldsymbol{y}=\boldsymbol{y}(\boldsymbol{w})$, where $\boldsymbol{y} \in \mathbb{R}^{n_{\mathrm{j}}-1}$ is a representation of the direction of the vector $\boldsymbol{w}$. The differential of this mapping takes the form $\mathrm{d} \boldsymbol{y}=\frac{\partial \boldsymbol{y}(\boldsymbol{w})}{\partial \boldsymbol{w}} \mathrm{d} \boldsymbol{w}$ and applying the chain rule results in

$$
\frac{\partial \boldsymbol{y}}{\partial \boldsymbol{w}} \mathrm{d} \boldsymbol{w}=\frac{\partial \boldsymbol{y}}{\partial \boldsymbol{w}} \frac{\partial \boldsymbol{w}}{\partial \boldsymbol{z}} \mathrm{d} \boldsymbol{z},
$$

where the $\left(n_{\mathrm{j}}-1\right) \times 2 n_{\mathrm{j}}$ Jacobian matrix $(\partial \boldsymbol{y} / \partial \boldsymbol{w})(\partial \boldsymbol{w} / \partial \boldsymbol{z})$ has full row rank. Note that the Jacobian matrix in (15) does not account for the constraints $\phi(z)=\mathbf{0}$ which represent an implicit relation between $\boldsymbol{q}_{\mathrm{j}}$ and $\boldsymbol{x}$. These constraints can be incorporated differentially by

$$
\left[\begin{array}{c}
\frac{\partial \boldsymbol{y}}{\partial \boldsymbol{w}} \mathrm{d} \boldsymbol{w} \\
\mathbf{0}
\end{array}\right]=\boldsymbol{J}(\boldsymbol{z}) \mathrm{d} \boldsymbol{z}
$$

where the Jacobian matrix $\boldsymbol{J}(\boldsymbol{z})=\left[\begin{array}{c}\frac{\partial \boldsymbol{y}}{\partial \boldsymbol{w}} \frac{\partial \boldsymbol{w}}{\partial \boldsymbol{z}} \\ \frac{\partial \boldsymbol{\phi}}{\partial \boldsymbol{z}}\end{array}\right]$ is now $\left(2 n_{\mathrm{j}}-\right.$ 1) $\times n_{\mathrm{j}}$ and therefore the linear system of equations (16) is still under-determined. The degree of freedom in the solution of (16) can be exploited to span one dimensional subspaces $^{3}$ where the motion along the oscillation mode $\boldsymbol{w}$ takes place. In case of mode matching it represents the vector space where changes in the configuration $\mathrm{d} \boldsymbol{q}_{\mathrm{j}}$ do not result in changes $\mathrm{d} \boldsymbol{w}$ of the oscillation mode $\boldsymbol{w}$. The remaining nullspace can be resolved by augmenting the Jacobian [21]: $\boldsymbol{J}_{\text {aug }}=\left(\begin{array}{c}\boldsymbol{J} \\ \boldsymbol{Z}\end{array}\right)$, where $\boldsymbol{Z} \in \mathbb{R}^{1 \times 2 n_{\mathrm{j}}}$ is a basis spanning the nullspace of $\boldsymbol{J}$ such that $\boldsymbol{J} \boldsymbol{Z}^{T}=\mathbf{0}$. Finally, the mode matching algorithm can be compactly described by the following formula:

$$
\boldsymbol{z}_{k+1}=\boldsymbol{z}_{k}+\gamma \boldsymbol{J}_{\text {aug }}\left(\boldsymbol{z}_{k}\right)^{-1}\left[\begin{array}{c}
\frac{\partial \boldsymbol{y}}{\partial \boldsymbol{w}}\left(\boldsymbol{w}_{\mathrm{des}}-\boldsymbol{w}_{k}\left(\boldsymbol{z}_{k}\right)\right) \\
\mathbf{0}_{n_{\mathrm{j}} \times 1} \\
\boldsymbol{Z}\left(\boldsymbol{z}_{k}\right)\left(\boldsymbol{z}_{\mathrm{des}}-\boldsymbol{z}_{k}\right)
\end{array}\right],
$$

where $\boldsymbol{z}_{\text {des }}$ is a desired configuration satisfying $\phi\left(\boldsymbol{z}_{\mathrm{des}}\right)=\mathbf{0}$ which "is kept in the nullspace of $\boldsymbol{J}$ " and $\gamma>0$ influences the convergence rate.

Remark 1: The closed-form computation (without finite differences) of the augmented Jacobian in (17) requires to compute the closed-form differentiation of the eigenvector $\boldsymbol{w}$ w.r.t. the variables $z$. This can be achieved using the procedure given in the Appendix II.

The modal matching algorithm and its intermediate results derived above, form the basis for the control approach proposed next.

\section{CONTROL APPROACH}

The basic idea of the control approach is to achieve directed (cyclic) jumping motions with minimal control action and thereby changing the original plant dynamics only to a minimum extent. The intention is to already achieve the desired behavior by means of the modal matching algorithm

\footnotetext{
${ }^{3}$ Note that there exist infinitely many possibilities to span the nullspace of $\boldsymbol{J}$.
}
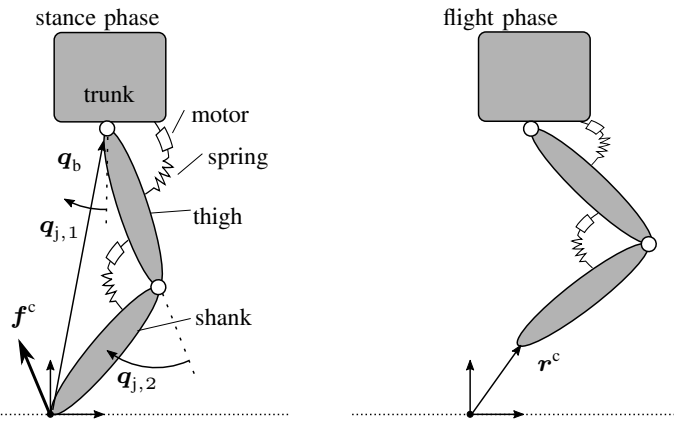

Fig. 2. Two segment leg setup considered for the derivation of the control approach. The stance phase (left) and the flight phase (right) are depicted.

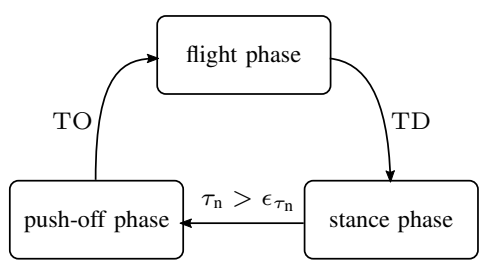

Fig. 3. Finite dynamics of the controller

derived in Sect. IV-C and use very low gain feedback control to regulate against model uncertainties and disturbances. In the following, we will introduce the control law exemplarily for a single two segment leg for which the base is free to translate in the sagittal plane (see, Fig. 2). At the end of the section, we will briefly discuss how the approach can be extended to legs including a foot and to multi-legged systems.

\section{A. Finite state machine}

The controller is embedded in a finite state machine which switches between control actions respectively feedback controls triggered based on state dependent events. As depicted in Fig. 3, the state machine has the following states:

- flight phase,

- stance phase,

- and push-off phase,

which also represent the phases of the controlled jumping motion. These phases are triggered by events which occur when the continuous system states hit the boundary of the corresponding switching manifold:

- The flight phase is triggerd by the takeoff event TO which occur when the normal component $f_{\mathrm{n}}^{\mathrm{c}}$ of the contact force defined in Fig. 2 becomes zero.

- The touchdown event TD triggers the stance phase when the foot hits the ground, i. e., when the distance between the contact point and the ground $r_{\mathrm{n}}^{\mathrm{c}}$ (see, Fig. 2) becomes zero.

- The generalized elastic force $\tau_{\mathrm{n}} \in \mathbb{R}$ (defined in (20)) reflecting the spring compression triggers the push-off phase when the threshold $\epsilon_{\tau_{\mathrm{n}}}$ is reached. Note that this phase change is part of the feedback control.

The following subsections derive the control action during these phases which lead to a continuing jumping cycle. 


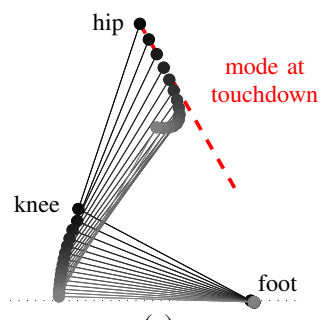

(a)

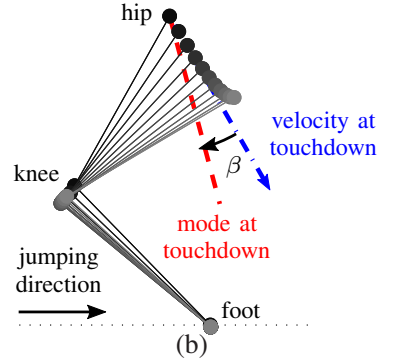

(b)
Fig. 4. (a) Deflection motion of the trunk due to the modal matching algorithm. The desired mode is represented as a dashed line. For perfect matching, the pivot point of the hip should move along the dashed line. (b) Definition of the angle of attack $\beta$, the angle between the touchdown velocity (dash-dotted blue direction) and the mode at touchdown (dashed red direction). Additionally, the effect of the angle of attack is shown. A tilting of the mode direction is achieved.

\section{B. Flight phase / foot placement}

In the first instance, the goal of our flight phase control is to reconfigure the leg such that the deflection motion after the touchdown is along the desired oscillation mode of the plant. The direction of the translational velocity of the base, i.e., $\alpha_{\mathrm{v}, \mathrm{TD}}=\operatorname{angle}\left(\dot{\boldsymbol{q}}_{\mathrm{b}}(\mathrm{TD})\right)$ is assumed to be known a priori. Note that $\alpha_{\mathrm{v}, \mathrm{TD}}$ can be predicted based on the takeoff velocity angle $\alpha_{\mathrm{v}, \mathrm{TO}}$ assuming a frictionless ballistic flight phase. In particular, $\alpha_{\mathrm{v}, \mathrm{TO}}$ can be predicted based on the joint velocity $\dot{\boldsymbol{q}}_{\mathrm{j}}$ just before the takeoff incorporating the stance phase constraint (3), Sect. III-C. Then, given $\alpha_{\mathrm{v}, \mathrm{TD}}$, the touchdown configuration can be computed based on the modal matching algorithm of Sect. IV-C for the desired oscillation mode $\boldsymbol{w}_{\mathrm{des}}=\boldsymbol{w}_{\mathrm{des}}\left(\alpha_{\mathrm{v}, \mathrm{TD}}\right)$.

For the two-segment leg example considered here, the degrees of freedom during contact are $n_{\mathrm{j}}=2$ such that the direction coordinate of the mode $y \in \mathbb{R}$ is scalar. Furthermore, the contact constraints can be solved explicitly, resulting in the mapping $\boldsymbol{q}_{\mathrm{b}}\left(\boldsymbol{q}_{\mathrm{j}}\right)$. As a consequence the augmented Jacobian of (17) simplifies to

$$
\boldsymbol{J}_{\mathrm{aug}}\left(\boldsymbol{q}_{\mathrm{j}}\right)=\left[\begin{array}{c}
\frac{\partial y(\boldsymbol{w})}{\partial \boldsymbol{w}} \frac{\partial \boldsymbol{w}\left(\boldsymbol{q}_{\mathrm{j}}\right)}{\partial \boldsymbol{q}_{\mathrm{j}}} \\
\boldsymbol{Z}\left(\boldsymbol{q}_{\mathrm{j}}\right)
\end{array}\right]
$$

where $\boldsymbol{Z}\left(\boldsymbol{q}_{\mathrm{j}}\right) \in \mathbb{R}^{1 \times 2}$ satisfies $\frac{\partial y(\boldsymbol{w})}{\partial \boldsymbol{w}} \frac{\partial \boldsymbol{w}\left(\boldsymbol{q}_{\mathrm{j}}\right)}{\partial \boldsymbol{q}_{\mathrm{j}}} \boldsymbol{Z}\left(\boldsymbol{q}_{\mathrm{j}}\right)^{T}=\mathbf{0}$, and the modal matching recursion (17) reduces to

$$
\boldsymbol{q}_{\mathrm{j}, k+1}=\boldsymbol{q}_{\mathrm{j}, k}+\gamma \boldsymbol{J}_{\mathrm{aug}}\left(\boldsymbol{q}_{\mathrm{j}, k}\right)^{-1}\left[\begin{array}{l}
\frac{\partial y}{\partial \boldsymbol{w}}\left(\boldsymbol{w}_{\mathrm{des}}-\boldsymbol{w}\left(\boldsymbol{q}_{\mathrm{j}, k}\right)\right) \\
\boldsymbol{Z}\left(\boldsymbol{q}_{\mathrm{j}, k}\right)\left(\boldsymbol{q}_{\mathrm{j}, \mathrm{des}}-\boldsymbol{q}_{\mathrm{j}, k}\right)
\end{array}\right]
$$

Fig. 4(a) depicts an example simulation result of the mode matching procedure. In the shown case, the deflection behavior is such that the motion is almost linear in the desired direction especially in the first part of the motion. Note that due to the mode matching procedure, the segmented leg behaves like a spring loaded telescopic leg as considered in the pogo-stick model of Raibert [9] or the spring loaded inverted pendulum model [10].

For a directed jumping motion, the touchdown and takeoff angles are of opposite sign. Therefore, the mode orientation needs to be tilted from the touchdown to the takeoff direction. The tilting is an effect which can be naturally achieved by means of the mode matching based reconfiguration. Therefore, we introduce a non-zero angle $\beta=y\left(\boldsymbol{q}_{\mathrm{j}}(\mathrm{TD})\right)-$ $\alpha_{\mathrm{v}, \mathrm{TD}}$ which we refer to as angle of attack (see, Fig. 4(b)). The angle of attack $\beta$ is a control input which indirectly influences the tilting momentum and can be used to control the horizontal jumping velocity. Since, the control variable of the proposed approach is the touchdown angle respectively takeoff angle, i.e., $\alpha_{\mathrm{v}, \mathrm{TD}}=-\alpha_{\mathrm{v}, \mathrm{TO}}$, we formulate the repetitive feedback law

$$
\beta(l+1)=\beta(l)-k_{\alpha}\left(\alpha_{\mathrm{v}, \mathrm{TO}}-\alpha_{\mathrm{v}, \mathrm{TO}}^{\mathrm{des}}\right)
$$

where $k_{\alpha}>0$ is a (low) feedback gain, $\alpha_{\mathrm{v}, \mathrm{TO}}^{\mathrm{des}}$ is the desired takeoff angle and $l$ is the iteration variable of the $l$-th jumping cycle.

Note that this concept generalizes also the foot placement algorithm of Raibert [9] as introduced for a telescopic leg to the case of a two-segment leg.

\section{Stance phase}

The stance phase is in charge of the two main tasks: (i) weight bearing and (ii) stabilizing the mode tilting. Thereby, the goal is that both tasks fit to our concept of natural dynamics exploitation. This is achieved by shaping the spring potential in coordinates based on the oscillation mode $\boldsymbol{w}$. Consider therefore the differential mapping based on the augmented Jacobian (18)

$$
\left[\begin{array}{c}
\dot{y} \\
v_{\mathrm{n}}
\end{array}\right]=\boldsymbol{J}_{\mathrm{aug}}\left(\boldsymbol{q}_{\mathrm{j}}\right) \dot{\boldsymbol{q}}_{\mathrm{j}}
$$

and its pull-back transformation (transforming generalized forces)

$$
\boldsymbol{\tau}_{\mathrm{j}}=\boldsymbol{J}_{\mathrm{aug}}\left(\boldsymbol{q}_{\mathrm{j}}\right)^{T}\left[\begin{array}{c}
\tau_{\mathrm{y}} \\
\tau_{\mathrm{n}}
\end{array}\right]
$$

where $v_{\mathrm{n}} \in \mathbb{R}$ is a velocity in the nullspace of the mapping $y=y\left(\boldsymbol{q}_{\mathrm{j}}\right)$. The generalized forces $\tau_{\mathrm{y}}$ and $\tau_{\mathrm{n}}$ are dual to $\dot{y}$ and $v_{\mathrm{n}}$, respectively.

Remark 2: At this point it is worth pointing out that, although the coordinate $y$ is based on the oscillation mode $\boldsymbol{w}$ which is a notion for linear dynamics, the mapping $y=y\left(\boldsymbol{q}_{\mathbf{j}}\right)$ is nonlinear and the considered coordinate transformation follows the strict rules of differential geometry as, e.g., explained in [22].

Using the above transformation, let us transform the joint torques $\boldsymbol{\tau}_{\mathrm{j}}\left(\boldsymbol{\theta}_{0}, \boldsymbol{q}_{\mathrm{j}}\right)$ defined by (2), where $\boldsymbol{\theta}_{0}:=\boldsymbol{q}_{\mathrm{j}}(\mathrm{TD})$ (constant during stance phase) is the result of the reconfiguration algorithm of Sect. V-B:

$$
\left[\begin{array}{c}
\tau_{\mathrm{y}}\left(\boldsymbol{\theta}_{0}, \boldsymbol{q}_{\mathrm{j}}\right) \\
\tau_{\mathrm{n}}\left(\boldsymbol{\theta}_{0}, \boldsymbol{q}_{\mathrm{j}}\right)
\end{array}\right]=\boldsymbol{J}_{\mathrm{aug}}\left(\boldsymbol{q}_{\mathrm{j}}\right)^{-T} \boldsymbol{\tau}_{\mathrm{j}}\left(\boldsymbol{\theta}_{0}, \boldsymbol{q}_{\mathrm{j}}\right) .
$$

In particular, $\boldsymbol{Z}\left(\boldsymbol{q}_{\mathrm{j}}\right)$ can be computed such that

$$
\tau_{\mathrm{n}}\left(\boldsymbol{\theta}_{0}, \boldsymbol{q}_{\mathrm{j}}\right)=\boldsymbol{Z}\left(\boldsymbol{q}_{\mathrm{j}}\right) \boldsymbol{\tau}_{\mathrm{j}}\left(\boldsymbol{\theta}_{0}, \boldsymbol{q}_{\mathrm{j}}\right)
$$

which represent the portion of the spring forces $\boldsymbol{\tau}_{\mathrm{j}}\left(\boldsymbol{\theta}_{0}, \boldsymbol{q}_{\mathrm{j}}\right)$ in the direction of the mode vector $\boldsymbol{w}$. This force implements the weight bearing task (i). 
The second task of the stance phase is to achieve stable transition from the direction of the touchdown mode to the direction of the push-off mode. Assuming, these directions are symmetric according to the vertical line, the stable transition can be implemented by the equilibrium position corresponding to the mode angle $y=y_{\text {des }}=0$. Note that the force $\tau_{\mathrm{y}}\left(\boldsymbol{\theta}_{0}, \boldsymbol{q}_{\mathrm{j}}\right)$ resulting from the springs in the joints (cf. (19)) does not implement the desired equilibrium $\tau_{\mathrm{y}}\left(\boldsymbol{\theta}_{0}, \boldsymbol{q}_{\mathrm{j}}\right)=0 \Longrightarrow y=y_{\text {des }}$. In particular, in the gravity free case, the equilibrium of the modal angle would be $y=\alpha_{\mathrm{v}, \mathrm{TD}}$ (i. e., the modal angle at touchdown resulting from the modal matching algorithm). To achieve the desired equilibrium, we artificially introduce a potential which produces the generalized force

$$
\tau_{\mathrm{y}}^{\mathrm{des}}=-k_{y}\left(y-y_{\mathrm{des}}\right)
$$

with $k_{y}>0$ being the stiffness of the potential. This control law stabilizes the mode tilting task (ii).

The complete stance phase control takes the form

$$
\boldsymbol{\tau}_{\mathrm{j}}^{\text {des }}=\boldsymbol{J}_{\text {aug }}\left(\boldsymbol{q}_{\mathrm{j}}\right)^{T}\left[\begin{array}{c}
-k_{y}\left(y-y_{\text {des }}\right) \\
\boldsymbol{Z}\left(\boldsymbol{q}_{\mathrm{j}}\right) \boldsymbol{\tau}_{\mathrm{j}}\left(\boldsymbol{\theta}_{0}, \boldsymbol{q}_{\mathrm{j}}\right)
\end{array}\right]
$$

Note that this control law only alters the elastic potential corresponding to the mode tilting coordinate $y$ and therefore changes the original plant dynamics only to a minimum extent.

Remark 3: As explained in Sect. III-B, the joint torque $\tau_{\mathrm{j}}$ is not an control input of the plant. To implement the control law (22), we invert the generalized elastic force function (2), Sect. III-B, (note that this is always possible due to the conditions on $\left.U\left(\boldsymbol{\theta}, \boldsymbol{q}_{\mathrm{j}}\right)\right)$ : $\boldsymbol{\theta}_{\mathrm{des}}=\boldsymbol{\tau}_{j}^{-1}\left(\boldsymbol{\tau}_{\mathrm{j}}^{\mathrm{des}}\right)+\boldsymbol{q}_{\mathrm{j}}$.

The procedure of partially changing the (visco-)elastic behavior in terms of task-coordinates has already been proposed in our previous work [18] for a Cartesian task. Here, we adapt the method to the case of modal coordinates and thereby we combine it with the novel modal matching approach.

\section{Push-off phase}

The push-off phase is triggered when the nullspace force $\tau_{\mathrm{n}}\left(\boldsymbol{\theta}_{0}, \boldsymbol{q}_{\mathrm{j}}\right)$ defined by (20) overshoots a certain threshold $\epsilon_{\tau_{\mathrm{n}}}$, following the concept of switching based limit cycle control as proposed in our previous work [23]. The control action of the push-off phase is a pure switching of the motor position in the direction of the instantaneous oscillation mode $\boldsymbol{w}\left(\boldsymbol{q}_{\mathrm{j}}(\mathrm{PO})\right)$, i.e.,

$$
\boldsymbol{\theta}_{\mathrm{des}}=\boldsymbol{\theta}(\mathrm{PO})+\frac{\left(\frac{\partial \boldsymbol{q}_{\mathrm{b}}\left(\boldsymbol{q}_{\mathrm{j}}\right)}{\partial \boldsymbol{q}_{\mathrm{j}}}\right)^{-1} \boldsymbol{w}\left(\boldsymbol{q}_{\mathrm{j}}(\mathrm{PO})\right)}{\left\|\left(\frac{\partial \boldsymbol{q}_{\mathrm{b}}\left(\boldsymbol{q}_{\mathrm{j}}\right)}{\partial \boldsymbol{q}_{\mathrm{j}}}\right)^{-1} \boldsymbol{w}\left(\boldsymbol{q}_{\mathrm{j}}(\mathrm{PO})\right)\right\|} \hat{\theta}
$$

where $\hat{\theta}>$ is a constant switching amplitude and PO denotes the time instance where the push-off phase is triggered. This control action is responsible for the energy input required to sustain the limit cycle.

It is worth mentioning that the limit cycle excitation considered here differs from our method proposed in [17].
In [17] the excitation is performed along the principal component of the motion which corresponds to an "average" of all local linear eigenmodes of the motion. Here, we excite the system along the instantaneous eigenmode. As such, push-off and deflection after the touchdown are modal and the transition between both phases is performed w.r.t. to modally based coordinates. Conceptually, in the presence of damping (which exists in every physical system), a modal excitation is the most efficient excitation. Since our concept exploits the oscillation mode in all phases of the jumping motion, the energy efficiency is potentially higher compared to motions which are less "modal".

\section{E. Additional degrees of freedom extension / multiple legs}

Here, we briefly discuss how the control approach presented above extends to the general case of additional degrees of freedom and multiple legs. Therefore, we subdivide the task coordinates in the components $\boldsymbol{x}=\left(\begin{array}{c}\boldsymbol{x}_{\mathrm{t}} \\ \boldsymbol{x}_{\mathrm{r}} \\ \boldsymbol{x}_{\mathrm{d}}\end{array}\right)$, where $\boldsymbol{x}_{\mathrm{t}}$ and $\boldsymbol{x}_{\mathrm{r}}$ represent the translation and rotation of the trunk, respectively, and the generalized force $\tau_{\mathrm{d}}$ dual to $\dot{\boldsymbol{x}}_{\mathrm{d}}$ represent the internal tension force due to multiple legs in contact. We may define the components of the oscillation mode accordingly: $\boldsymbol{w}=\left(\begin{array}{c}\boldsymbol{w}_{\mathrm{t}} \\ \boldsymbol{w}_{\mathrm{r}} \\ \boldsymbol{w}_{\mathrm{d}}\end{array}\right)$. If the jumping task requires a purely translational motion, we may match to a desired mode of the form $\boldsymbol{w}_{\mathrm{des}}=\left(\begin{array}{c}\boldsymbol{w}_{\mathrm{t}}\left(\boldsymbol{\alpha}_{\mathrm{v}, \mathrm{TD}}\right) \\ \boldsymbol{w}_{\mathrm{r}}=\mathbf{0} \\ \boldsymbol{w}_{\mathrm{d}}=\mathbf{0}\end{array}\right)$. The stance phase control can be performed for the translational part of the dynamics (corresponding to $\boldsymbol{x}_{\mathrm{t}}$ ), while the rotational motion $\dot{x}_{\mathrm{r}}$ and the tension force $\tau_{\mathrm{d}}$ need to be regulated to zero. A comprehensive analysis on the coupling between the tasks will be part of future work.

\section{SIMULATION RESULTS}

This section conducts simulations ${ }^{4}$ of a system as depicted in Fig. 2 to evaluate the performance of the controller introduced in Sect. V. Fig. 5 shows the compression of the springs along the modal direction as well as the transition to the push-off phase, the flight phase and the foot placement based on modal matching. Fig. 6 validates the steady-state behavior of the approach by showing the limit cycle of the hip angle, knee angle, and vertical trunk position. Finally, Fig. 7 depicts the convergence behavior of the takeoff angle, which is a control variable of the repetitive low gain control.

The stability and robustness properties of the closed-loop jumping system have been additionally analyzed using the numerical Poincaré return map method. Since the control system dynamics is hybrid, the formulation of the Poincare return map for hybrid systems as proposed in [25, Chapt. 4] has been utilized. It is worth mentioning that the assumptions

\footnotetext{
${ }^{4}$ The differential equations of the closed-loop system has been integrated using a variable step solver of MATLAB/Simulink. The contact dynamics has been incooporated in the differential equations using a compliant contact model as described by [24].
} 


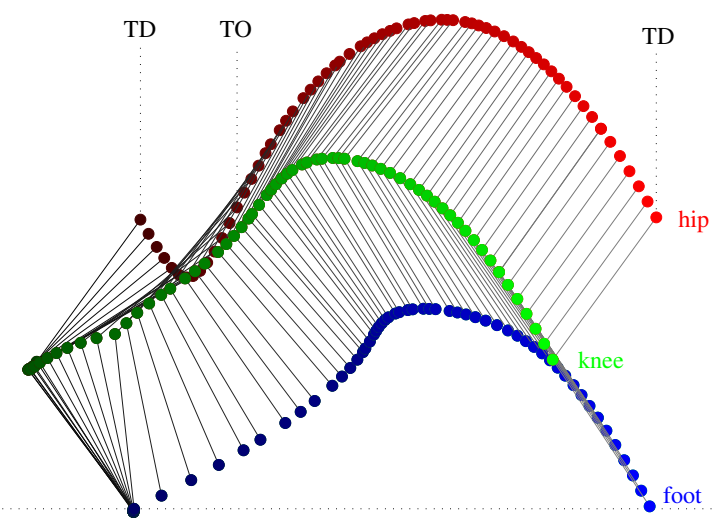

Fig. 5. Motion of a complete jumping cycle between two consecutive touchdown (TD) events of the two segment, compliantly actuated robot model. Red points correspond to the hip, green points to the knee and blue points to the foot. The horizontal positions of the hip at TD and takeoff (TO) are marked by vertical dotted lines. A symmetric motion of the hip (red dots) between the first TD and TO can be observed.

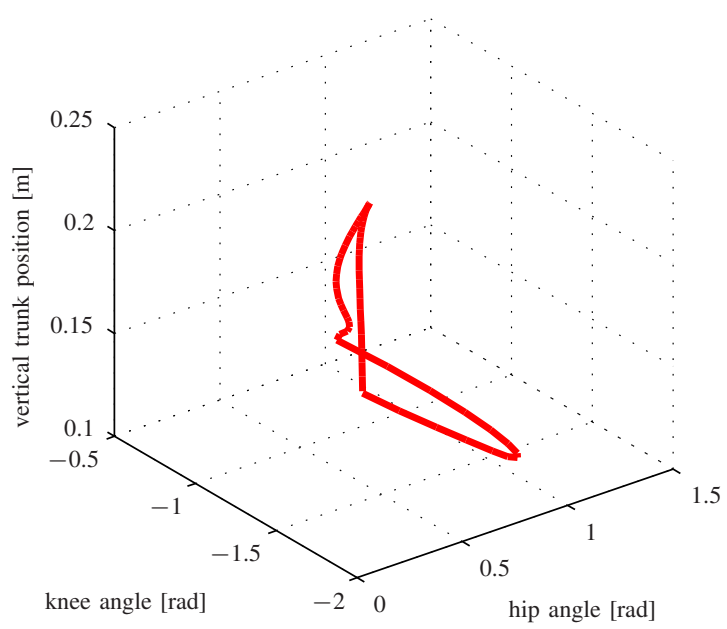

Fig. 6. Limit cylce hip angle vs. knee angle vs. vertical trunk position.

of [25, Theorem 4.1 and 4.3] have been checked and are satisfied, but due to space limitations only the results of the analysis are presented in Fig. 8 and 9. Fig. 8 depicts the convergence behavior for a perturbation of the initial conditions with respect to the fixed-point. Fig. 9 depicts the convergence behavior for a perturbation of the model parameters used in the feedback control. Both figures represent the Poincaré return map considering the takeoff condition as Poincaré section. The evaluation of the Jacobian matrices of the Poincaré return maps reveals that the jumping system has an exponentially stable fixed-point even in the case of parameter uncertainties.

\section{CONCLUSION}

The concept of modal matching and its application to natural jumping control introduced here, bridges the gap between performance and efficiency. While our previous and existing approaches satisfy only one of these controller design goals, i.e., forced desired oscillations or natural resulting oscillations, the methodology introduced here achieves both design criteria. As a result, modal matching generalizes the

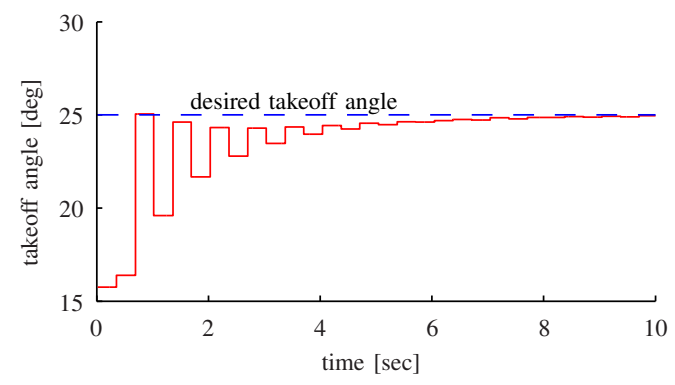

Fig. 7. Convergence of the takeoff angle. Solid line represents the current takeoff angle and dashed line the desired takeoff angle.

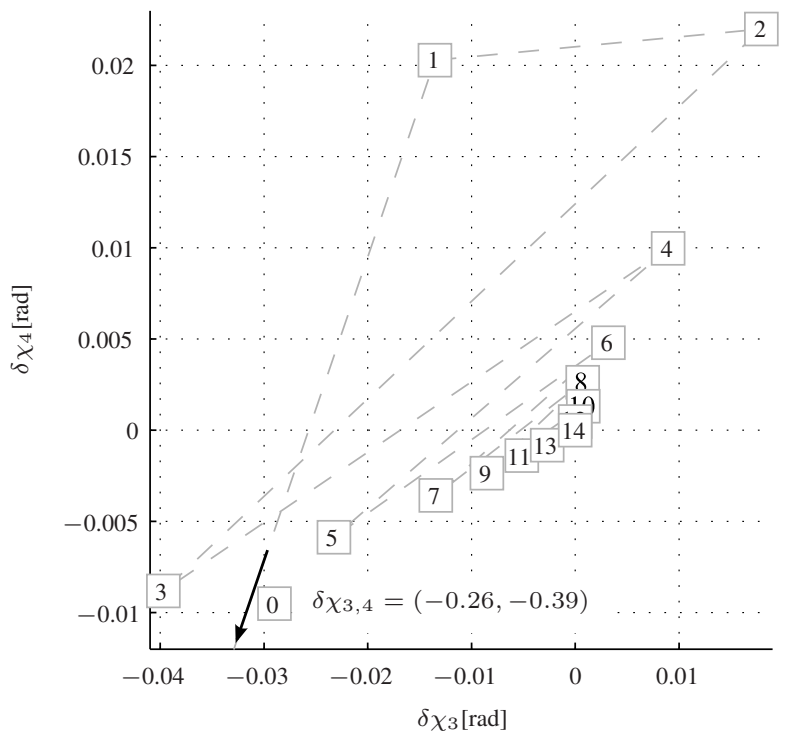

Fig. 8. Graphical representation of the Poincaré return map corresponding to the exponentially stable fixed-point (eigenvalues magnitude of the Jacobian linearization are strictly less than one) $\chi^{\star}=$ $(0,-0.02,0.77,-0.89,0.64,1.41,-19.55,40.48)$. The deviation from the fixed-point $\delta \chi=\chi_{k}-\chi^{\star}$ are plotted for the states corresponding to the joint configuration variables $\boldsymbol{q}_{\mathrm{j}}$.

spring-mass and pogo-stick model (which are very basic to locomotion) to the case of articulated, elastic multi-body systems.

\section{APPENDIX I}

\section{COMPONENTS OF THE CONSTRAINED INERTIA AND} STIFFNESS MATRIX

Using the notations $\boldsymbol{\Phi}_{\mathrm{jb}}=\left(\frac{\partial \phi}{\partial \boldsymbol{q}_{\mathrm{j}}}\right)^{-1} \frac{\partial \phi}{\partial \boldsymbol{q}_{\mathrm{b}}}, \boldsymbol{\Phi}_{\mathrm{jd}}=$ $\left(\frac{\partial \phi}{\partial \boldsymbol{q}_{\mathrm{j}}}\right)^{-1} \frac{\partial \phi}{\partial d}$ for the constrained Jacobian matrices, the components of the closed-loop inertia matrix in (9), $\bar{M}=$ $\left[\begin{array}{ll}\overline{\boldsymbol{M}}_{\mathrm{bb}} & \overline{\boldsymbol{M}}_{\mathrm{bd}} \\ \overline{\boldsymbol{M}}_{\mathrm{bd}}^{T} & \overline{\boldsymbol{M}}_{\mathrm{dd}}\end{array}\right]$, can be computed by $\overline{\boldsymbol{M}}_{\mathrm{bb}}=\boldsymbol{M}_{\mathrm{bb}}$ $\boldsymbol{M}_{\mathrm{bj}} \boldsymbol{\Phi}_{\mathrm{jb}}-\boldsymbol{\Phi}_{\mathrm{jb}}^{T} \boldsymbol{M}_{\mathrm{bj}}^{T}+\boldsymbol{\Phi}_{\mathrm{jb}}^{T} \boldsymbol{M}_{\mathrm{jj}} \boldsymbol{\Phi}_{\mathrm{jb}}, \overline{\boldsymbol{M}}_{\mathrm{bd}}=-\boldsymbol{M}_{\mathrm{bj}} \boldsymbol{\Phi}_{\mathrm{jd}}+$ $\boldsymbol{\Phi}_{\mathrm{jb}}^{T} \boldsymbol{M}_{\mathrm{jj}} \boldsymbol{\Phi}_{\mathrm{jd}}$, and $\overline{\boldsymbol{M}}_{\mathrm{dd}}=\boldsymbol{\Phi}_{\mathrm{jd}}^{T} \boldsymbol{M}_{\mathrm{jj}} \boldsymbol{\Phi}_{\mathrm{jd}}$. Analogously, the components of the closed-loop stiffness matrix, $\bar{K}=$ $\left[\begin{array}{cc}\overline{\boldsymbol{K}}_{\mathrm{bb}} & \overline{\boldsymbol{K}}_{\mathrm{bd}} \\ \overline{\boldsymbol{K}}_{\mathrm{bd}}^{T} & \overline{\boldsymbol{K}}_{\mathrm{dd}}\end{array}\right]$, can be computed by $\overline{\boldsymbol{K}}_{\mathrm{bb}}=\boldsymbol{\Phi}_{\mathrm{jb}}^{T} \boldsymbol{K} \boldsymbol{\Phi}_{\mathrm{jb}}$, $\overline{\boldsymbol{K}}_{\mathrm{bd}}=\boldsymbol{\Phi}_{\mathrm{jb}}^{T} \boldsymbol{K} \boldsymbol{\Phi}_{\mathrm{jd}}, \overline{\boldsymbol{K}}_{\mathrm{dd}}=\boldsymbol{\Phi}_{\mathrm{jd}}^{T} \boldsymbol{K} \boldsymbol{\Phi}_{\mathrm{jd}}$. 


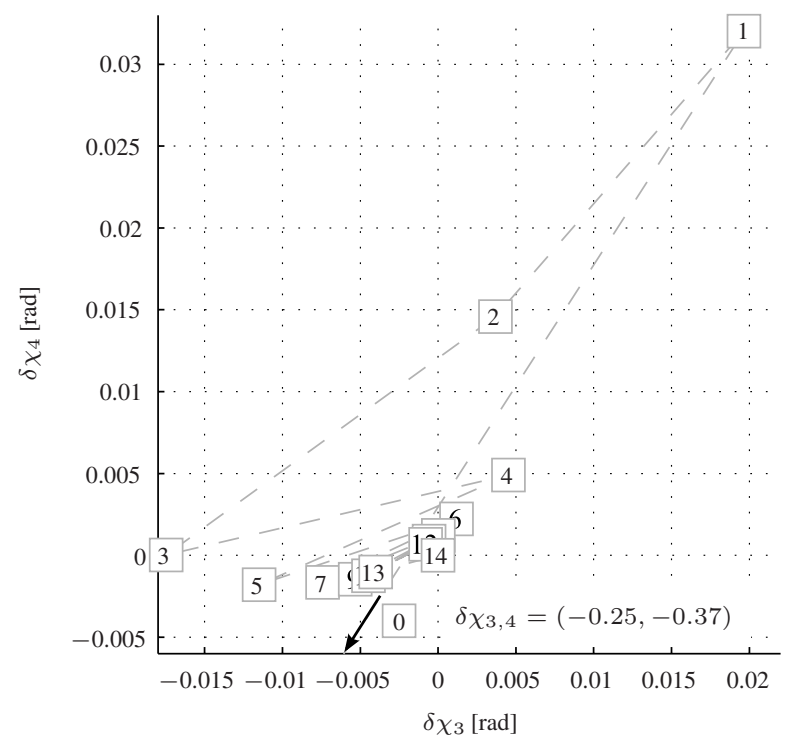

Fig. 9. Graphical representation of the Poincaré return map corresponding to the exponentially stable fixed-point (eigenvalues magnitude of the Jacobian linearization are strictly less than one) $\chi^{\star}=$ $(0,-0.02,0.78,-0.90,0.68,1.45,-19.68,41.34)$. The initial conditions equal to the one corresponding to the simulation in Fig. 8, but the model parameters considered in the modal matching controller are perturbed (joint stiffness and trunk inertia of the model deviate by $10 \%$, sign alternately). Note that the parameter deviation leads to a slightly changed but also exponentially stable fixed-point.

\section{APPENDIX II}

\section{DIFFERENTIATION OF EIGENVECTORS}

Given are two symmetric matrices $\boldsymbol{K}, \boldsymbol{M} \in \mathbb{R}^{m \times m}$ with $M$ positive definite. Consider the generalized eigenvalue problem (11), (12), where $\overline{\boldsymbol{K}} \triangleq \boldsymbol{K}$ and $\overline{\boldsymbol{M}} \triangleq \boldsymbol{M}$. Assume that all quantities in the above equations are functions of the variables $\boldsymbol{z}=\left(z_{1}, z_{2}, \ldots, z_{n}\right)^{T}$. The goal is to compute the Jacobian matrix $\frac{\partial \boldsymbol{w}}{\partial \boldsymbol{z}}=\left(\begin{array}{llll}\frac{\partial \boldsymbol{w}}{\partial z_{1}} & \frac{\partial \boldsymbol{w}}{\partial z_{2}} & \cdots & \frac{\partial \boldsymbol{w}}{\partial z_{n}}\end{array}\right)$. Therefore, we show how to compute $\partial \boldsymbol{w} / \partial z_{i}$. We derive both sides of (11) and rearrange the equation as

$$
(\boldsymbol{K}-\lambda \boldsymbol{M}) \frac{\partial \boldsymbol{w}}{\partial z_{i}}=\frac{\partial \lambda}{\partial z_{i}} \boldsymbol{M} \boldsymbol{w}-\left(\frac{\partial \boldsymbol{K}}{\partial z_{i}}-\lambda \frac{\partial \boldsymbol{M}}{\partial z_{i}}\right) \boldsymbol{w}
$$

Pre-multiplying (24) by $\boldsymbol{w}^{T}$ from the left and taking into account that $\boldsymbol{w}^{T}(\boldsymbol{K}-\lambda \boldsymbol{M})=\mathbf{0}$ (cf. (11)), leads to the derivative of $\lambda$ as

$$
\frac{\partial \lambda}{\partial z_{i}}=\frac{\boldsymbol{w}^{T}\left(\frac{\partial \boldsymbol{K}}{\partial z_{i}}-\lambda \frac{\partial \boldsymbol{M}}{\partial z_{i}}\right) \boldsymbol{w}}{\boldsymbol{w}^{T} \boldsymbol{M} \boldsymbol{w}} .
$$

To compute $\partial \boldsymbol{w} / \partial z_{i},(25)$ is substituted in (24):

$$
(\boldsymbol{K}-\lambda \boldsymbol{M}) \frac{\partial \boldsymbol{w}}{\partial z_{i}}=\left(\frac{\boldsymbol{M} \boldsymbol{w} \boldsymbol{w}^{T}}{\boldsymbol{w}^{T} \boldsymbol{M} \boldsymbol{w}}-\boldsymbol{I}\right)\left(\frac{\partial \boldsymbol{K}}{\partial z_{i}}-\lambda \frac{\partial \boldsymbol{M}}{\partial z_{i}}\right) \boldsymbol{w} .
$$

Since the matrix $(\boldsymbol{K}-\lambda \boldsymbol{M})$ is singular by definition, one equation in (26) has to be replaced by the derivative of (12), $2 \boldsymbol{w}^{T} \frac{\partial \boldsymbol{w}}{\partial z_{i}}=0$ such that the resulting system of linear equations becomes regular.

\section{REFERENCES}

[1] R. M. Alexander, "Three uses for springs in legged locomotion," International Journal of Robotics Research, vol. 9, no. 2, pp. 53-61, 1990.

[2] J. W. Hurst, The role and implementation of compliance in legged locomotion. ProQuest, 2008.

[3] D. J. Braun, M. Howard, and S. Vijayakumar, "Exploiting variable stiffness in explosive movement tasks," in Robotics: Science and Systems, 2011.

[4] D. Braun, F. Petit, F. Huber, S. Haddadin, P. van der Smagt, A. AlbuSchaffer, and S. Vijayakumar, "Optimal torque and stiffness control in compliantly actuated robots," in Proc. IEEE/RSJ Int. Conf. on Intelligent Robots and Systems. IEEE, 2012.

[5] S. Haddadin, F. Huber, and A. Albu-Schäffer, "Optimal control for exploiting the natural dynamics of variable stiffness robots," in IEEE Int. Conf. on Robotics and Automation, 2012.

[6] D. Braun, F. Petit, F. Huber, S. Haddadin, P. van der Smagt, A. AlbuSchaffer, and S. Vijayakumar, "Robots driven by compliant actuators: Optimal control under actuation constraints," Robotics, IEEE Transactions on, vol. 29, no. 5, pp. 1085-1101, Oct 2013.

[7] G. A. Pratt and M. M. Williamson, "Series elastic actuators," in Intelligent Robots and Systems 95.'Human Robot Interaction and Cooperative Robots', Proceedings. 1995 IEEE/RSJ International Conference on, vol. 1. IEEE, 1995, pp. 399-406.

[8] A. Albu-Schäffer, O. Eiberger, M. Grebenstein, S. Haddadin, C. Ott, T. Wimböck, S. Wolf, and G. Hirzinger, "Soft robotics," Robotics \& Automation Magazine, IEEE, vol. 15, no. 3, pp. 20-30, 2008

[9] M. H. Raibert, Legged Robots That Balance. The MIT Press, 1986.

[10] R. Blickhan, "The spring-mass model for running and hopping," Journal of Biomechanics, vol. 22, pp. 1217-1227, 1989.

[11] A. Seyfarth, H. Geyer, M. Gnther, and R. Blickhan, "A movement criterion for running." Journal of Biomechanics, vol. 35, no. 5, pp. 649-55, 2002.

[12] H. Geyer, A. Seyfarth, and R. Blickhan, "Compliant leg behavior explains basic dynamics of walking and running," Proceedings of the Royal Society B, vol. 273, pp. 2861-2867, Nov. 2006.

[13] J. Rummel, Y. Blum, H. M. Maus, C. Rode, and A. Seyfarth, "Stable and robust walking with compliant legs," in Proc. of IEEE Int. Conf. on Robotics and Automation, 2010, pp. 5250-5255.

[14] J. Pratt, P. Dilworth, and G. Pratt, "Virtual model control of a bipedal walking robot," in IEEE Int. Conf. on Robotics and Automation, 1997, pp. 193-198.

[15] I. Poulakakis and J. W. Grizzle, "The spring loaded inverted pendulum as the hybrid zero dynamics of an asymmetric hopper," IEEE Transactions on Automatic Control, vol. 54, no. 8, pp. 1779-1793, 2009

[16] M. Hutter, D. Remy, M. A. Höpflinger, and R. Siegwart, "Slip running with an articulated robotic leg," in Proc. of IEEE/RSJ Int. Conf. on Intelligent Robots and Systems, 2010, pp. 4934-4939.

[17] D. Lakatos, M. Görner, F. Petit, A. Dietrich, and A. Albu-Schäffer, "A modally adaptive control for multi-contact cyclic motions in compliantly actuated robotic systems," in Proc. IEEE/RSJ Int. Conf. on Intelligent Robots and Systems, 2013, pp. 5388-5395.

[18] D. Lakatos, G. Garofalo, A. Dietrich, and A. Albu-Schäffer, "Jumping control for compliantly actuated multilegged robots," in IEEE Int. Conf. on Robotics and Automation, 2014, pp. 4562-4568.

[19] M. W. Spong, "Modeling and control of elastic joint robots," Transactions of the ASME: Journal of Dynamic Systems, Measurement, and Control, vol. 109, pp. 310-319, 1987.

[20] P. Kokotovic, H. Khalil, and J. O'Reilly, Singular Perturbation Methods in Control: Analysis and Design. Academic Press, London, 1986.

[21] J. Park, W. Chung, and Y. Youm, "On dynamical decoupling of kinematically redundant manipulators," in IEEE/RSJ International Conference on Intelligent Robots and Systems, 1999, pp. $1495-1500$.

[22] T. Frankel, The Geometry of Physics: An Introduction, 2nd ed. Cambridge University Press, 2003.

[23] D. Lakatos, F. Petit, and A. Albu-Schäffer, "Nonlinear oscillations for cyclic movements in human and robotic arms," IEEE Transactions on Robotics, vol. 30, no. 4, pp. 865-879, 2014.

[24] M. Azad and R. Featherstone, "Modeling the contact between a rolling sphere and a compliant ground plane," ACRA, Brisbane, Australia, 2010.

[25] E. R. Westervelt, J. W. Grizzle, C. Chevallereau, J. H. Choi, and B. Morris, Feedback Control of Dynamic Bipedal Robot Locomotion. CRC Press, 2007. 